\title{
Iterated local search for the team orienteering problem with time windows.
}

Pieter Vansteenwegen ${ }^{*}$, , Wouter Souffriau ${ }^{1,2}$, Greet Vanden Berghe ${ }^{2}$, Dirk Van Oudheusden $^{1}$

${ }^{1}$ Centre for Industrial Management, Katholieke Universiteit Leuven, Celestijnenlaan 300A - bus 2422, 3001 Leuven, Belgium. Tel: +32 16322567. Fax: +3216322986.

\{Pieter.Vansteenwegen; Dirk.VanOudheusden\}@cib.kuleuven.be

${ }^{2}$ Information Technology, Katholieke Hogeschool Sint-Lieven, Gebroeders

Desmetstraat 1, 9000 Gent, Belgium.

\{Wouter.Souffriau; Greet.VandenBerghe\}@kahosl.be

*Corresponding author 


\begin{abstract}
$\underline{\text { Abstract }}$
In the Team Orienteering Problem with Time Windows (TOPTW) a set of locations is given, each with a score, a service time and a time window. The goal is to determine a fixed number of routes, limited in length, that visit, at the right time, some locations and maximise the sum of the collected scores. This paper describes a simple, fast and effective iterated local search meta-heuristic to solve the TOPTW. An insert step is combined with a shake step to escape local optima. The specific shake step implementation produces a heuristic that performs very well on a large set of instances with up to 288 locations. The obtained results have an average gap with the optimal solution of only $1.8 \%$ for instances with only a single route and $2.1 \%$ for instances with three to twenty routes.
\end{abstract}

Keywords: Iterated Local Search, Team Orienteering Problem with Time Windows, Meta-Heuristics 


\section{Introduction}

In the Orienteering Problem with Time Windows (OPTW) a set of $n$ locations is given: each location $i=1, \ldots, n$ is assigned a score $S_{i}$, a service or visiting time $T_{i}$ and a time window $\left[O_{i}, C_{i}\right]$. The starting point (location 1) and the end point (location $n$ ) of every tour are fixed. The time $t_{i j}$ needed to travel from location $i$ to $j$ is known for all locations. Not all locations can be visited since the available time is limited to a given time budget $T_{\max }$. The OPTW goal is to determine a single route, limited by $T_{\max }$, that visits some of the locations during their appropriate time windows, and that maximises the total collected score. Each location can be visited at most once. The Team Orienteering Problem with Time Windows (TOPTW) is an OPTW where the goal is to determine $m$ routes, each limited to $T_{\max }$, that maximises the total collected score.

The OP [1] is also known as the selective travelling salesperson problem [2], the maximum collection problem [3] and the bank robber problem [4]. Furthermore, the OP can be formulated as a special case of the resource constrained TSP [5], a TSP with profits [6] or as a resource constrained elementary shortest path problem [7].

Many (T)OP applications are described in the literature: the sport game of orienteering [8], the home fuel delivery problem [9], athlete recruiting from high schools [3], routing technicians to service customers [10], etc. Usually, most of these applications also require time windows and, as a consequence, the TOPTW model can be applied to many real life situations. The application we are most interested in is related to what we call the Personalised Electronic Tourist Guide (PET). The PET, a handheld device, is, among other things, aware of the owner's user profile, values of tourist attractions, and trip restrictions. The device is 
capable of suggesting, at short notice, a (near) optimal combination of interesting attractions in a city and a route between them, taking into account the weather, opening hours, crowded places, etc. Typically, the PET has to solve Tourist Trip Design Problems (TTDP) [11]. The TOPTW can be seen as a simplified TTDP.

The best published heuristics for the TOP are described in Archetti et al. [12] and Vansteenwegen et al. [13]. These algorithms, however, cannot solve (T)OP with time windows efficiently. All of them apply local search moves that become useless when time windows have to be considered.

Boussier et al. [14] describe an exact solution method to solve small and medium size TOP instances to optimality, as well as selective vehicle routing problems with time windows, a generalisation of the TOPTW. A detailed and extended review of many algorithms for the OP and the TOP is provided in Tang and Miller-Hooks [10].

Many articles have been published regarding vehicle routing with time windows. Yet, only four articles deal with the orienteering problem with time windows [15, $16,17,18]$ and only one [14] was found about (a generalization of) the team orienteering problem with time windows.

Kantor and Rosenwein [15] were the first to solve the OPTW. They first describe a straightforward insertion heuristic. The location with the highest ratio "score over insertion time" is inserted into the tour, without violating any of the time windows. Secondly, a depth-first search tree algorithm is proposed that constructs partial tours, using the insertion heuristic and beginning in the start location. Partial routes are abandoned if they become infeasible or if they are unlikely to yield the best total score. Righini and Salani [7] apply bi-directional dynamic programming to solve OPTW to optimality. Starting forward from the start point 
and backwards from the end point, current states are extended by adding an extra location at the end. Forward and backward states are matched if feasible and dominance tests are applied to record only non-dominated states. Decremental state space relaxation [18] is used to reduce the number of states to be explored. The data sets of Righini and Salani and the optimal results they calculated will be used to validate the heuristic of this paper. Bar-Yehuda et al. [16] also mention the OPTW, but only for the special cases where all locations have the same score and locations are located on a line or in the Euclidean plane. They do not consider time limits on the tour duration.

In the next Section a rigorous problem definition is given, in Section 3 the heuristic is described in detail and in Section 4 experimental results are presented. Conclusions and further work are discussed in Section 5.

\section{Mathematical problem definition}

Based on the notation introduced above, the team orienteering problem with time windows can be formulated as an integer program $\left(x_{i j d}=1\right.$ if, in route $d$, a visit to location $i$ is followed by a visit to location $j, 0$ otherwise; $y_{i d}=1$ if location $i$ is visited in route $d, 0$ otherwise; $a_{i d}=$ the arrival time at location $i$ in route $d ; s_{i d}=$ the start of the service at location $i$ in route $d ; M$ a large constant):

$$
\begin{aligned}
& \text { (0) } \operatorname{Max} \sum_{d=1}^{m} \sum_{i=2}^{n-1} S_{i} y_{i d} \\
& \text { (1) } \sum_{d=1}^{m} \sum_{j=2}^{n-1} x_{1 j d}=\sum_{d=1}^{m} \sum_{i=2}^{n-1} x_{i n d}=m
\end{aligned}
$$



(2) $\sum_{i=1}^{n-1} x_{i k d}=\sum_{j=2}^{n} x_{k j d}=y_{k d}$
$(k=2, \ldots, n-1 ; d=1, \ldots, m)$
(3) $s_{i d}+T_{i}+c_{i j}-a_{j d} \leq M\left(1-x_{i j d}\right)$
$(i, j=1, \ldots, n ; d=1, \ldots, m)$
(4) $\quad \sum_{d=1}^{m} y_{k d} \leq 1$
$(k=2, \ldots, n-1)$
(5) $\quad \sum_{i=1}^{n-1}\left(T_{i} y_{i d}+\sum_{j=2}^{n} c_{i j} x_{i j d}\right) \leq T_{\max }$
$(d=1, \ldots, m)$
(6) $O_{i} \leq s_{i d}$
$(i=1, \ldots, n ; d=1, \ldots, m)$
(7) $\quad a_{i d} \leq s_{i d}$
$(i=1, \ldots, n ; d=1, \ldots, m)$
(8) $s_{i d}+T_{i} \leq C_{i}$
$(i=1, \ldots, n ; d=1, \ldots, m)$
(9) $x_{i j d}, y_{i d} \in\{0,1\}$
$(i, j=1, \ldots, n ; d=1, \ldots, m)$

The objective function (0) maximises the total collected score. Constraint (1) guarantees that all tours start in location 1 and end in location $n$. Constraints (2) and (3) ensure the connectivity and timeline of each tour. Constraints (4) ensure that every location is visited at most once and constraints (5) limit the time budget. Constraints (6) and (7) make sure that, if the arrival at a location happens before it opens, the service itself can only start when the time window opens. Constraints (8) guarantee that each service is finished before the time window closes.

Mathematical formulations of the TOP (without time windows) can be found in Butt and Cavalier [3], Tang and Miller-Hooks [10] and Boussier et al. [14]. 


\section{$\underline{3 \text { Iterated local search heuristic }}$}

The TOPTW is a highly constrained problem and very difficult to solve. Since Golden et al. [9] prove that the OP is NP-hard, it is highly unlikely that the TOPTW can be solved to optimality within polynomial time. For many practical applications and especially for the PET, it is required to solve TOPTW with high quality in only a few seconds. Gendreau et al. [19] discuss a few reasons why it is so difficult to design high-quality heuristics for the (T)OP. The score of a location and the distance to reach it are independent and often in opposition to each other. This makes it very difficult to select the locations that will be part of the optimal solution. Therefore, simple construction and improvement heuristics may direct the algorithm in undesirable directions as they do not scrutinise sufficiently large parts of the solution landscape and because wrong decisions are unsatisfactorily corrected. The time windows further complicate the solution process.

Nevertheless, a straightforward and fast iterated local search heuristic performing very well on the available data sets, has been developed. The heuristic combines an insertion step and a shaking step to escape local optima.

\section{$\underline{3.1 \text { Insertion Step }}$}

The insertion step tries to add new visits to a tour, one by one. Before an extra visit can be inserted in a tour, it should be verified that all visits scheduled after the insertion place still satisfy their time window. In order to develop a fast heuristic, a quick evaluation of each possible insert move is necessary. Therefore, checking all other visits on their feasibility would take too much time. This can be avoided by recording MaxShift for each included location. MaxShift is defined as the maximum time the service completion of a given visit can be delayed, without 
making any visit infeasible. Another useful concept is Wait, i.e. the waiting time in case the arrival at a location takes place before the time window is open. The service itself can only start when the time window opens. If the arrival takes place during the time window, Wait equals zero. MaxShift of location $i$ is equal to the sum of Wait and MaxShift of the next location $i+1$, unless MaxShift is limited by its own time windows $\left(C_{i}\right)$.

$$
\begin{aligned}
& \text { MaxShift }_{i}=\min \left[C_{i}-\left(s_{i}+T_{i}\right), \text { Wait }_{i+1}+\text { MaxShift }_{i+1}\right] \\
& \text { Wait }_{i}=\max \left[0, O_{i}-a_{i d}\right]
\end{aligned}
$$

For a feasible insertion of a new visit $j$ between visits $i$ and $k$, the total time consumption $\left(\right.$ Shift $\left._{i}\right)$ of visit $j$ should be limited to the sum of Wait $_{k}$ and MaxShift M $_{k}$ of visit $k$. This gives the following formula to check feasibility:

$$
\text { Shift }_{j}=c_{i j}+\text { Wait }_{j}+T_{j}+c_{j k}-c_{i k} \leq \text { Wait }_{k}+\text { MaxShift }_{k}
$$

Obviously, service $j$ should also fit the time window of location $j$.

For each visit that can be inserted, the Shift is determined. The visit with the highest ratio "square of location score over Shift" will be selected for insertion. The score is applied to the power two because, due to time windows and waiting, the time consumption (Shift) becomes less relevant than score for adding new visits to the tour. The ratio of the score over Shift could also be used, but the obtained results are clearly worse, as will be shown in Section 4. 
All steps mentioned above do not require much computation time since only very simple and quick evaluations are used, thanks to the MaxShift recording.

After insertion, all other visits should be updated. Visits after the insertion require an update of the waiting time (Wait), the arrival time $(a)$, the start of the service (s) and MaxShift. Every time a visit possesses a waiting time, the time shift for the start of that service and all following services will be reduced by this waiting time.

The following formulas are used to update the visits after the insertion position, when visit $j$ is inserted between $i$ and $k$ :

$$
\begin{aligned}
& \text { Shift }_{j}=c_{i j}+\text { Wait }_{j}+T_{j}+c_{j k}-c_{i k} \\
& \text { Wait }_{k^{*}}=\max \left[0, \text { Wait }_{k}-\text { Shift }_{j}\right] \\
& a_{k^{*}}=a_{k}+\text { Shift }_{j} \\
& \text { Shift }_{k}=\max \left[0, \text { Shift }_{j}-\text { Wait }_{k}\right] \\
& s_{k^{*}}=s_{k}+\text { Shift }_{k} \\
& \text { MaxShift }_{k^{*}}=\text { MaxShift }_{k}-\text { Shift }_{k}
\end{aligned}
$$

Shift $_{k}$ and the same formulas are then used to update the visit after $k$, and so on, until Shift is reduced to zero.

Visits before the insertion may require an update of MaxShift, using formula (10) mentioned above. By way of illustration, a small example is discussed (See Figures 1 and 2).

\section{Figure 1}

Figure 2 
It is feasible to insert $j\left(O_{j}=13, C_{j}=31, T_{j}=5\right)$ between $i\left(O_{i}=1, C_{i}=33, T_{i}=5\right)$ and $k$ $\left(O_{k}=17, C_{k}=36, T_{k}=8\right):$

$$
\text { Shift }_{j}=c_{i j}+\text { Wait }_{j}+T_{j}+c_{j k}-c_{i k}=1+2+4+3-3 \leq 4+8=\text { Wait }_{k}+\text { MaxShift }_{k}
$$

Because visit $j$ is inserted, $k$ and $l$ need to be updated. The arrival at location $k$ $\left(a_{k}=13\right)$ is delayed by the total time consumption of the insertion (Shift $\left.t_{j}=7\right)$, thus $a_{k^{*}=20}$. Due to Wait $_{k}$ (4) the time shift is reduced to 3 (=Shift $\left.{ }_{k}\right)$ and the start of the service itself $\left(s_{k}=17\right)$ is only delayed by $\operatorname{Shift}_{k}\left(s_{\left.k^{*}=20\right) \text {. Furthermore, MaxShift }}\right.$ is reduced by Shift $t_{k}$. Since Wait $_{l}(3)$ is equal to or bigger than Shift $t_{k}, s_{l}$ and MaxShift are unchanged; only Wait $_{l^{*}}(0)$ and $a_{l^{*}}$ (30) need to be updated. Any visit scheduled after $l$ will not be affected by the insertion of $j$. Applying the abovementioned formulas:

$$
\begin{aligned}
& \text { Wait }_{k^{*}}=\max \left[0, \text { Wait }_{k}-\text { Shift }_{j}\right]=\max [0,4-7]=0 \\
& a_{k^{*}}=a_{k}+\text { Shift }_{j}=13+7=20 \\
& \text { Shift }_{k}=\max \left[0, \text { Shift }_{j}-\text { Wait }_{k}\right]=\max [0,7-4]=3 \\
& S_{k^{*}}=s_{k}+\text { Shift }_{k}=17+3=20 \\
& \text { MaxShift }_{k^{*}}=\text { MaxShift }_{k}-\text { Shift }_{k}=8-3=5 \\
& \text { Wait }_{l^{*}}=\max \left[0, \text { Wait }_{l}-\text { Shift }_{k}\right]=\max [0,3-3]=0 \\
& a_{l^{*}}=a_{l}+\text { Shift }_{k}=27+3=30 \\
& \text { Shift }_{l}=\max \left[0, \text { Shift }_{k}-\text { Wait }_{l}\right]=\max [0,3-3]=0
\end{aligned}
$$


For visits $h$ and $i$, MaxShift need to be updated. For visit $i$ in the example, MaxShift $i^{*}$ is equal to the sum of Wait $_{j^{*}}$ and MaxShift $j^{*}$. Due to the time window of location $h$, the end of service $h$ can only be delayed by one time unit. Thus MaxShift $_{h *}$ remains equal to 1 and the insertion of visit $j$ has no impact on a visit before visit $h$.

\subsection{Shake Step}

The Shake Step is used to escape local optima. During this step, one or more visits will be removed. The Shake Step uses two parameters as input, the first one indicates how many consecutive visits to remove in each tour (NumberToRemove), the second indicates the place in each tour to start the removing process (PositionStartRemove). If during the removal the end location is reached, the removal continues after the start location. After the removal, all visits following the removed visits are shifted to the beginning of the tour as much as possible, in order to avoid unnecessary waiting. These visits should be updated similarly to the updating in the Insert Step. For the visits before the removed visits, only MaxShift should be updated.

If the Shake Step is applied on the abovementioned example (Figure 2), with PositionStartRemove and NumberToRemove both equal to two, visits $i$ and $j$ are removed and the result will be h-k-l (Figure 3). Visit $k$ can start three time units earlier after waiting ten units. Visit $l$ cannot start earlier due to the time window; the arrival is thus earlier, but the start of the service remains. MaxShift ${ }_{h}$ is not changed due to the time window of location $h$. Due to the time windows of location $h$ and $l$, no visits before visit $h$ or after visit $l$ change because of this removal. The large values Wait $_{k}$ and MaxShift can $_{\text {cow }}$ be used to visit another 
(combination of) location(s) characterised by a higher score than locations $i$ and $j$ combined.

Figure 3

\section{$\underline{3.3 \text { Heuristic }}$}

Figure 4 presents the heuristic's flow diagram. The heuristic starts with empty tours and initialises all the parameters of the Shake Step to one. The heuristic runs through a loop until no improvements are identified after a fixed number of iterations. Firstly the Insert Step is used repeatedly until a local optimum is reached. If this solution is better than the incumbent solution, the solution is recorded and NumberToRemove is reset to one for the next Shake Step. Secondly Shake Step is applied. After each Shake Step, PositionStartRemove is increased by the value NumberToRemove and NumberToRemove is increased by one for the next Shake Step. If PositionStartRemove equals the size of the smallest tour, this size is subtracted to determine the new position. If NumberToRemove equals 30 , it is reset to one. This heuristic resembles what Lourenço et al. [20] call Iterated Local Search (ILS): iteratively, a sequence of local search solutions is built up, instead of repeating random trials of the local search. ILS has shown before to work well on problems with time windows [21, 22].

By using the shake parameters as described above, other visits are removed during every Shake Step and during the entire procedure, most likely, every visit is removed at least once. This proves to be an excellent technique to solve the commonly known problems mentioned by Gendreau et al. [19] when using simple improvement heuristics. The entire solution space is now better explored and 
earlier taken wrong decisions are corrected. This property is enhanced by the fact that the heuristic always continues the search from the current solution, it never returns to the best found solution to continue. This is called iterated local search with a random walk acceptance criterion [20].

The maximum number of locations to remove $\left(n /\left(3^{*} m\right)\right)$ and the maximum number of iterations without improvement (150) are the only parameters to predetermine in this heuristic. For the maximum number of locations to remove a percentage of $n / m$ (number of locations/number of routes) is used. Changing this percentage, from $n / m$ to $n /\left(5^{*} m\right)$, has no significant effect on the quality of the results or computation times. Furthermore, it appeared that further increasing the maximum number of iterations without improvement does not significantly improve the obtained results and only causes longer computation times.

\section{Figure 4}

\section{Experimental results:}

\section{$\underline{4.1 \text { Test instances }}$}

The heuristic was tested on the available test instances and compared with published results. Righini and Salani [17] created 58 instances for the OPTW using Solomon's data set of vehicle routing problems with time windows and ten problems of multi-depot vehicle routing problems of Cordeau, Gendreau and Laporte. Important to notice is that in the original data sets the time windows $\left(O_{i}\right.$, $\left.C_{i}\right)$ are defined only as bounds for the start of the visit $\left(s_{i}\right)$. As a consequence the end of the visit $\left(l_{i}\right)$ can take place after the time window has closed. In our formulation the whole visit should happen during the time window. This is more 
realistic in the context of tourism where time windows correspond to opening hours. In order to compare results, the time windows of the original data sets are changed, the closing times are extended by the visit duration to correspond with the formulation $\left(O_{i}, C_{i}+T_{i}\right)$.

For the OP with multiple routes, the TOP, only test sets are available for instances without time windows $[12,14]$. Since instances with time windows (TOPTW) are different from instances without time windows, TOP test sets cannot be used to verify the performance of the ILS heuristic. For instance, the well-known two-opt move [23] is indispensable to obtain high-quality results for the TOP, but due to the time windows, it cannot be used to solve the TOPTW efficiently. Obviously, the ILS algorithm, without a two-opt move, would not perform well in solving TOP instances. Furthermore, Boussier et al. [14] report on experimental results for the selective vehicle routing problem with time windows, a generalisation of the TOPTW. Compared to the TOPTW, two extra constraints are added, one related to the capacity of each vehicle and one related to the travel distance. The ILS heuristic in this paper cannot take these extra constraints into account.

Therefore, two new sets of 68 and 39 TOPTW instances are designed based on the data sets of Righini and Salani. The first data set uses all the instances from Righini and Salani (68), but with the number of tours equal to two instead of one. A commercial solver (CPLEX 11) could only solve four of these instances to optimality if the calculation time is six hours. This illustrates the difficulty of the instances. As a consequence, two times the optimal score of the original instance is used as a benchmark for the obtained total score. This is obviously an overestimation since the optimal result can never be higher, but it can be much lower than this number. 
The second new data set uses the original instances from Solomon (29) and Cordeau, Gendreau and Laporte (10), with the number of tours equal to the number of vehicles. With this number of tours it should be possible to visit every location and hence the optimal result equals the sum of all scores. However, since these instances are now seen as orienteering problems, the algorithm does not have the knowledge that all locations can be visited. It tries to select as many visits as possible and to design a feasible route between them. Since these instances have up to twenty tours they should be regarded as cases of rather intractable TOPTWs. Obviously, the difficulty of the instances is not only related to the number of locations that can be visited in each tour, but also to the number of tours that can be generated. All computations were carried out on a personal computer Intel Pentium 4 with $1.80 \mathrm{GHz}$ processor and $1 \mathrm{~GB}$ Ram. Righini and Salani did their calculations on a comparable PC Pentium 4 with $1.6 \mathrm{GHz}$ processor and $512 \mathrm{MB}$ Ram.

\section{$\underline{4.2 \text { Results }}$}

Tables 1 and 2 compare the scores obtained by the newly proposed heuristic with the optimal results of the OPTW instances. Column one and two give the instance's name, the number of locations and the time budget. The third column presents the score obtained by the ILS heuristic and the fourth column exhibits the optimal solution calculated by Righini and Salani. The gap between the optimal solution and the ILS solution, in column five, is stated as a percentage. In column six the number of visited locations of the solution is presented and the computation time in the last column is expressed in seconds. 


\section{Table 1}

\section{Table 2}

The average gap with the optimal solution for all these instances is only $1.8 \%$. In the worst case, with 288 locations, the gap is $9.4 \%$ and in 32 of the 68 cases the optimal solution is obtained. The average calculation time for Solomon's instances is only 0.4 seconds, compared to more than 400 seconds with the fastest method of Righini and Salani. Furthermore, it is clear that the computation time is correlated with the number of locations visited by the solution.

In the ILS heuristic the ratio "square of location score Shift" is applied to determine the next visit to insert in the tour. If, instead, the ratio "score over Shift" is used, the results are worse. For the instances mentioned above, the average gap with the optimal solution becomes $3.4 \%$, instead of $1.8 \%$. The worst solution has a gap of $27.8 \%$ instead of $9.4 \%$. To prove the key contribution of the Shake Step, the instances are also solved without using the Shake Step. In this case the average gap is $9.2 \%$ and the worst gap is $30.6 \%$.

In Tables 3 and 4 the results for TOPTW with $m$ equal to two are presented. In this case, column four does not represent the optimal solution but only an upper bound, as mentioned above. Even compared with this upper bound, the average gap remains acceptable. Four instances were solved to optimality by a commercial solver (CPLEX 11): c101_50 (490), r101_50 (246), r105_50 (282) and rc101_50 (360). The average gap between the ILS results and these optimal solutions is $1.6 \%$

Table 3

Table 4 
In Tables 5 and 6 scores are shown that are obtained for the new data sets of TOPTW with up to twenty tours. A column is added with the number of routes for each instance. The optimal score is the sum of the score of all locations. The average gap with the optimal solution for all these instances is only $2.1 \%$. The worst score has a gap of $10.0 \%$. The quality of these results is almost equal to the quality of the results of instances with one tour. Of course, the computation time for these very complicated instances is much longer, due to the many tours that have to be constructed and improved. Yet, the average computation time is still many times shorter than the computation time of Righini and Salani to solve the instances with only one tour. All test results are summarised in Table 7.

\section{Table 5}

Table 6

Table 7

Finally, it should be noticed that in this case always the exact distance between two locations is used, where Righini and Salani always rounded down the distance to the first (Solomon) or second (Cordeau, Gendreau and Laporte) decimal. Obviously, rounding down distances makes it sometimes possible to add an extra visit.

\section{Conclusions and further work}

The new heuristic solves the Team Orienteering Problem with Time Windows (TOPTW) fast and effectively. An Insert Step is applied every iteration to 
improve the incumbent solution. A Shake Step is implemented in a specific manner to successfully escape local optima. The heuristic performs very well on a large set of instances with up to 288 locations. For 68 instances with one route the average gap with the optimal solution is only $1.8 \%$, and the worst gap is $9.4 \%$. The average computation time is 1.0 seconds. For 39 instances with a range of three to twenty routes, the average gap is $2.1 \%$ and the worst gap is $10.0 \%$. Clearly, the average computation time of 17.9 seconds is longer here, but still very small considering the complexity of the instances. As far as we know, this is the first heuristic to tackle TOPTW. New test sets are generated and our results can be used as a benchmark for further research.

In the future, this heuristic can be tailored to solve realistic tourist trip design problems. Some improvements are possible due to the specificity of time windows in tourist applications. Typically, most tourist attractions will be open all day and only the opening and closing times will be slightly different and, as a result, time windows will largely overlap. In this situation, adding two-opt moves [23] to the heuristic could be beneficial and significantly reduce the travel time. Another promising move is the insertion of two or more activities simultaneously. Such moves can be imbedded in a variable neighbourhood search framework [24].

\section{Acknowledgements}

Dr. Vansteenwegen is a post doctoral research fellow of the "Fonds Wetenschappelijk Onderzoek- Vlaanderen (FWO)". 


\section{$\underline{\text { References: }}$}

[1] Tsiligirides T. Heuristic Methods Applied to Orienteering. Journal of the Operational Research Society 1984; 35; 797-809.

[2] Laporte G, Martello S. The Selective Travelling Salesman Problem. Discrete applied mathematics 1990;26; 193-207.

[3] Butt S, Cavalier T. A heuristic for the multiple tour maximum collection problem. Computers \& Operations Research 1994; 21; 101-111.

[4] Arkin E, Mitchell J, Narasimhan G. Resource-Constrained Geometric Network Optimization. Proceedings of the $14^{\text {th }}$ ACM Symposium on Computational Geometry 1998; 307-316.

[5] Pekny J, Miller D. An Exact Parallel Algorithm for the Resource Constrained Travelling Salesman Problem With Application to Scheduling With an Aggregate Deadline. Proceedings of the 1990 ACM Annual Conference on Cooperation.

[6] Feillet D, Dejax P, Gendreau M. Travelling Salesman Problems With Profits. Transportation Science 2005; 39; 188-205.

[7] Righini G, Salani M. Decremental state space relaxation strategies and initialization heuristics for solving the orienteering problem with time windows with dynamic programming. Accepted for publication: Computers \& Operations Research 2008a.

[8] Chao I, Golden B, Wasil E. A fast and effective heuristic for the Orienteering Problem. European Journal of Operational Research 1996; 88; 475-489.

[9] Golden B, Levy L, Vohra R. The orienteering problem. Naval Research Logistics 1987; 34; 307-318. 
[10] Tang H, Miller-Hooks E. A Tabu search heuristic for the team orienteering problem. Computers \& Operations Research 2005; 32; 1379-1407.

[11] Vansteenwegen P, Van Oudheusden D. The Mobile Tourist Guide: An OR Opportunity. OR Insights 2007; 20(3); 21-27.

[12] Archetti C, Hertz A, Speranza M. Metaheuristics for the team orienteering problem. Journal of Heuristics 2007; 13; 49-76.

[13] Vansteenwegen P, Souffriau W, Vanden Berghe G, Van Oudheusden D. A Guided Local Search Metaheuristic for the Team Orienteering Problem. Accepted for publication: European Journal of Operational Research 2008; doi:10.1016/j.ejor.2008.02.037.

[14] Boussier S, Feillet D, Gendreau M. An exact algorithm for the team orienteering problem. 4OR 2007; 5; 211-230.

[15] Kantor M, Rosenwein M. The Orienteering Problem with Time Windows. The Journal of the Operational Research Society 1992; 43(6); 629-635.

[16] Bar-Yehuda R, Even G, Shahar S. On Approximating a Geometric PrizeCollecting Travelling Salesman Problem with Time Windows. Journal of Algorithms 2005; 55; 76-92.

[17] Righini G, Salani M. Dynamic programming for the orienteering problem with time windows. Technical Report 91 2006, Dipartimento di Tecnologie dell'Informazione, Universita degli Studi Milano, Crema, Italy.

[18] Righini G, Salani M. New dynamic programming algorithms for the Resource Constrained Elementary Shortest Path. Accepted for publication: Networks 2008b. 
[19] Gendreau M, Laporte G, Semet F. A Tabu Search heuristic for the undirected selective travelling salesman problem. European Journal of Operational Research 1998; 106; 539-545.

[20] Lourenço H, Martin O, Stützle T. Iterated Local Search. In: Glover F, Kochenberger G (Eds.). Handbook of Metaheuristics. Dordrecht: Kluwer Academic Publishers, 2003. p. 321-353.

[21] Ibaraki T, Imahori S, Kubo M, Masuda T, Uno T, Yagiura M. Effective Local Search Algorithms for Routing and Scheduling with General Time-Window Constraints. Transportation Science 2005; 39(2); 206-232.

[22] Hashimoto H, Yagiura M, Ibaraki T. An iterated local search algorithm for the time-dependent vehicle routing problem with time windows. Discrete Optimization 2008; 5; 434-456.

[23] Lin S. Computer Solutions of the Traveling Salesman Problem. Bell Syst. Tech. J. 1965; 44; 2245-2269.

[24] Hansen P, Mladenovic N. Variable neighbourhood search: Principles and applications. European Journal of Operational Research 2001; 130; 449-467. 


\section{$\underline{\text { Figure captions }}$}

Figure 1: Tour $h-i-k-l$.

Figure 2: Tour $h-i-j-k-l$.

Figure 3: Tour $h-k-l$.

Figure 4: Flow diagram of the new heuristic. 